\title{
Peak-to-Average Power-Ratio Reduction for OFDM Systems Based on Method of Conditional Probability and Coordinate Descent Optimization
}

\author{
Y. J. Kou, W.-S. Lu, and A. Antoniou \\ Department of Electrical and Computer Engineering, University of Victoria \\ P.O. Box 3055, Victoria, B.C., Canada V8W 3P6 \\ Email: \{ykou,wslu\}@ece.uvic.ca, aantoniou@ieee.org
}

\begin{abstract}
A new constellation extension technique for peak-toaverage power-ratio reduction (PAPR) in orthogonal frequencydivision multiplexing systems is proposed. Two new algorithms for PAPR reduction are developed by applying the so-called method of conditional probability (MCP) and coordinate descent optimization (CDO). Our simulations demonstrate that the proposed algorithms outperform several existing algorithms and the performance can be further improved by combining the MCP, CDO, and the selective mapping algorithms.
\end{abstract}

\section{INTRODUCTION}

Orthogonal frequency-division multiplexing (OFDM) has recently been used for data transmission in a number of communication systems [1][2]. A major problem associated with OFDM is its large peak-to-average power-ratio (PAPR) which renders system performance sensitive to distortion introduced by nonlinear devices, e.g., power amplifiers (PAs). Recently, a number of algorithms [3]-[5] have been proposed to reduce the PAPR of the transmit signal before it enters the PAs.

In this paper, a new constellation is proposed whereby the data are represented either by points in the original constellation or by extended points. Two new algorithms are then developed by applying the so-called method of conditional probablity (MCP) [6]-[7] and coordinate descent optimization (CDO) [8], which can be used to find the optimal representation of the OFDM signal. Design examples are presented which demonstrate that significant reduction in PAPR as well as reduced computational effort can be achieved by the proposed algorithms over several existing algorithms. The performance of the proposed algorithms can be further improved by combining them with the selective mapping (SLM) algorithm [3].

\section{PAPR REDUCTION VIA CONSTELlation EXTENSION}

Consider an $N$-subcarrier OFDM transmitter as illustrated in Fig. 1, where $\mathrm{S} / \mathrm{P}, \mathrm{P} / \mathrm{S}$, and $\mathrm{D} / \mathrm{A}$ represent serialto-parallel, parallel-to-serial, and digital-to-analog converter, respectively, and the block labeled as "Amp." represents a power amplifier. A 16-QAM modulation is adopted for each subcarrier and its constellation is shown in Fig. 2a. The proposed constellation extension scheme is illustrated in Fig. $2 b$ where any data point with a value greater than or equal to 4 and less than 12 can be represented by a pair of two possible constellation points. For example, the data point $D_{k}=11$ (or 1011 in binary form) can be represented by either $X_{k}^{0}=-1-3 j$ or $X_{k}^{1}=-1+5 j$ where the superscript of $X_{k}$ is used to identify which constellation point is selected to represent $D_{k}$, i.e., $X_{k}^{0}$ indicates that an exterior point of the conventional constellation is used to represent $D_{k}$; on the other hand, $X_{k}^{1}$ indicates that a corresponding extended point is used to represent $D_{k}$. For the purpose of comparison, the constellation extension scheme proposed in [5] is shown in Fig. 2c.

The 16-QAM modulated symbol $X_{k}$ is referred to as the subsymbol at the $k$ th subcarrier, and vector $\boldsymbol{X}=$ $\left[\begin{array}{lll}X_{0} & \cdots & X_{N-1}\end{array}\right]$ is referred to as the OFDM symbol. The discrete complex baseband representation of the $n$th sample of the OFDM symbol is given by

$$
x_{n}=\frac{1}{N} \sum_{k=0}^{N-1} s_{k} X_{k} e^{\frac{j 2 \pi k n}{N}} \text { for } n=0, \ldots, N-1
$$

If we let $\mathbf{x}=\left[\begin{array}{lll}x_{0} & \cdots & x_{N-1}\end{array}\right]^{T}$, the PAPR of signal $\mathbf{x}$ can be defined as

$$
\operatorname{PAPR}=\frac{\|\mathbf{x}\|_{\infty}^{2}}{\mathcal{E}\left[\|\mathbf{x}\|_{2}^{2}\right] / N}
$$

where $\mathcal{E}[\cdot]$ denotes expectation. Our objective is to obtain an optimal representation of the data points by either the exterior or the extended points such that the PAPR of the OFDM symbol $\boldsymbol{X}$ is minimized. For the sake of a fair comparison with other PAPR-reduction algorithms, the peak power of the transmit signal will be used as a performance measure in the computer simulations presented in Sec. IV. Denoting the number and index set of subcarriers which are applicable to constellation extension as $K$ and $\mathcal{I}=\left\{i_{1}, i_{2}, \ldots, i_{K}\right\}$, respectively, and letting $\overline{\boldsymbol{X}}=\left[X_{i_{1}} \cdots X_{i_{K}}\right]^{T}$, the PAPRreduction problem can be formulated as

$$
\begin{aligned}
& \underset{\overline{\boldsymbol{X}}}{\operatorname{minimize}} \max _{0 \leq n \leq N-1}\left|\sum_{k=0, k \in \mathcal{I}}^{N-1} X_{k} e^{\frac{j 2 \pi k n}{N}}\right| \\
& \text { subject to: } X_{k} \in\left\{X_{k}^{0}, X_{k}^{1}\right\} \quad \text { for } k \in \mathcal{I}
\end{aligned}
$$


If we let

$$
Y_{k}=\frac{X_{k}^{0}+X_{k}^{1}}{2} \text { and } Z_{k}=\frac{X_{k}^{0}-X_{k}^{1}}{2} \text { for } k \in \mathcal{I}
$$

then we have

$$
X_{k}^{0}=Y_{k}+Z_{k} \text { and } X_{k}^{1}=Y_{k}-Z_{k} \quad \text { for } k \in \mathcal{I}
$$

where the variables in (3) and (4) are complex. If we define

$$
c_{n}= \begin{cases}\operatorname{Re}\left[\sum_{k=0, k \notin I}^{N-1} X_{k} e^{\frac{j 2 \pi k n}{N}}+\right. & \\ \left.\sum_{k=1}^{K} Y_{i_{k}} e^{\frac{j 2 \pi i_{k} n}{N}}\right] & 0 \leq n \leq N-1 \\ \operatorname{Im}\left[\sum_{k=0, k \notin I}^{N-1} X_{k} e^{\frac{j 2 \pi k(n-N)}{N}}+\right. & \\ & \left.\sum_{k=1}^{K} Y_{i_{k}} e^{\frac{j 2 \pi i_{k}(n-N)}{N}}\right] \quad N \leq n \leq 2 N-1\end{cases}
$$

and

$$
d_{n k}= \begin{cases}\operatorname{Re}\left[Z_{i_{k}} e^{\frac{j 2 \pi i_{k} n}{N}}\right] & 0 \leq n \leq N-1 \\ \operatorname{Im}\left[Z_{i_{k}} e^{\frac{j 2 \pi i_{k}(n-N)}{N}}\right] & N \leq n \leq 2 N-1\end{cases}
$$

then the problem in (3) can be relaxed to

$$
\begin{aligned}
& \underset{\boldsymbol{s}}{\operatorname{minimize}} \max _{0 \leq n \leq 2 N-1}\left|c_{n}+\sum_{k=1}^{K} s_{k} d_{n k}\right| \\
& \text { subject to: } s_{k} \in\{1,-1\} \text { for } k=1, \ldots, K
\end{aligned}
$$

where the variables involved are real. It can be shown that the solution of the problem in (6) can be regarded as a good approximation of the solution of the problem in (3). For this reason, a reasonable suboptimal solution to the PAPRreduction problem in (3) can be obtained by solving the problem in (6).

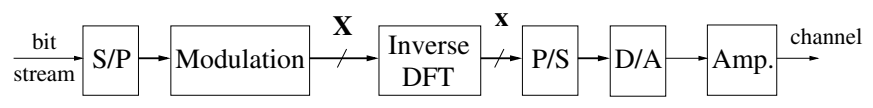

Fig. 1. Block diagram of a typical OFDM transmitter.

\section{PAPR-REDUCTION ALgORITHMS}

The minimax optimization problem in (6) is an integer programming problem which can be solved by using the MCP along with the pessimistic estimator reported in [6][7] or by using the CDO reported in [8].

\section{A. Algorithm Based on Method of Conditional Probability}

Consider sign vector $s=\left[s_{1} \cdots s_{K}\right]$ where $s_{1}, \ldots, s_{K}$ are treated as random variables which can assume the values of 1 or -1 with equal probability. Let $A_{n}^{\lambda}$ be the event that $\left|c_{n}+\sum_{k=1}^{K} d_{n k} s_{k}\right| \geq \lambda$ and let $\operatorname{Pr}\left(A_{n}^{\lambda}\right)$ be the probability that event $A_{n}^{\lambda}$ occurs. For the problem in (6), a pessimistic estimator is defined as an upper bound of the conditional probability $\operatorname{Pr}\left(A_{n}^{\lambda} \mid s_{1}, \ldots, s_{j}\right)$ and can be characterized by

$$
\operatorname{Pr}\left(A_{n}^{\lambda} \mid s_{1}, \ldots, s_{j}\right) \leq U_{n}\left(\lambda, s_{1}, \ldots, s_{j}\right)
$$

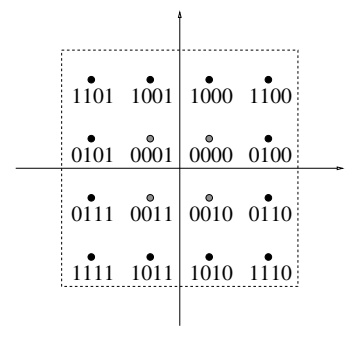

(a)

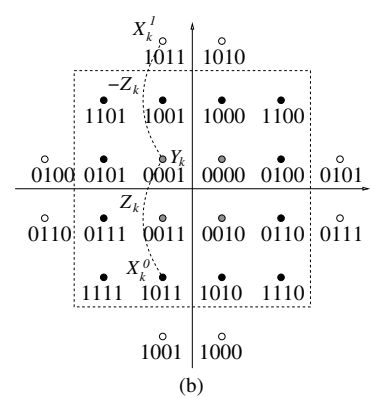

Fig. 2. (a) 16-QAM constellation with Gray code bit mapping. (b) An 8point extension scheme for 16-QAM constellation. (c) A 12-point extension scheme for 16-QAM constellation.

for $j=1, \ldots, K$, where the following condition is satisfied

$$
\begin{gathered}
\min _{s_{j} \in\{1,-1\}} \sum_{n=0}^{2 N-1} U_{n}\left(\lambda, s_{1}, \ldots, s_{j-1}, s_{j}\right) \\
\leq \sum_{n=0}^{2 N-1} U_{n}\left(\lambda, s_{1}, \ldots, s_{j-1}\right)
\end{gathered}
$$

Let us assume that $\lambda$ is chosen such that

$$
\sum_{n=0}^{2 N-1} U_{n}(\lambda)<1
$$

where $U_{n}(\lambda)$ denotes the upper bound of $\operatorname{Pr}\left(A_{n}^{\lambda}\right)$ with all the components of $\mathrm{s}$, i.e., $s_{1}, \ldots, s_{K}$ treated as random variables. If the first component of the optimal sign vector is taken to be $s_{1}^{\star}=1$, a suboptimal sign vector $s^{\star}$ can be obtained sequentially as

$$
\begin{aligned}
s_{j}^{\star}= & \arg \left[\min _{s_{j} \in\{1,-1\}} \sum_{n=0}^{2 N-1} U_{n}\left(\lambda, s_{1}^{\star}, \ldots, s_{j-1}^{\star}, s_{j}\right)\right] \\
= & -\operatorname{sign}\left[\sum_{n=0}^{2 N-1} U_{n}\left(\lambda, s_{1}^{\star}, \ldots, s_{j-1}^{\star}, 1\right)\right. \\
& \left.-\sum_{n=0}^{2 N-1} U_{n}\left(\lambda, s_{1}^{\star}, \ldots, s_{j-1}^{\star},-1\right)\right]
\end{aligned}
$$

for $j=2, \ldots, K$. Consequently, we have

$$
\begin{aligned}
& \sum_{n=0}^{2 N-1} U_{n}\left(\lambda, s_{1}^{\star}, \ldots, s_{j-1}^{\star}, s_{j}^{\star}\right) \\
& \leq \sum_{n=0}^{2 N-1} U_{n}\left(\lambda, s_{1}^{\star}, \ldots, s_{j-1}^{\star}\right)
\end{aligned}
$$


which in conjunction with (7), (8), and (9) implies that

$$
\begin{aligned}
\sum_{n=0}^{2 N-1} \operatorname{Pr}\left(A_{n}^{\lambda} \mid s_{1}^{\star}, \ldots, s_{K}^{\star}\right) & \leq \sum_{n=0}^{2 N-1} U_{n}\left(\lambda, s_{1}^{\star}, \ldots, s_{K}^{\star}\right) \\
& <1
\end{aligned}
$$

With the vector $s^{\star}$ known, the probability $\operatorname{Pr}\left(A_{n}^{\lambda} \mid s_{1}^{\star}\right.$, $\left.\ldots, s_{K}^{\star}\right)$ for each $n$ is either zero or one. Hence, it can be inferred from (12) that $\operatorname{Pr}\left(A_{n}^{\lambda} \mid s_{1}^{\star}, \ldots, s_{K}^{\star}\right)=0$ for $n=$ $0, \ldots, 2 N-1$, which means that $\left|c_{n}+\sum_{k=1}^{K} s_{k}^{\star} d_{n k}\right|<\lambda$. In other words, the sign vector $s^{\star}=\left[s_{1}^{\star} \cdots s_{K}^{\star}\right]$ obtained using (10) can be regarded as a suboptimal solution for which the objective function in the problem in (6) is guaranteed to be smaller than $\lambda$.

In what follows, a pessimistic estimator is derived based on the Chernoff bound [9] which can be described by the inequality

$$
\operatorname{Pr}(Y \geq \delta) \leq e^{-\gamma \delta} E\left(e^{\gamma Y}\right)
$$

where $\gamma$ is a nonnegative parameter to be optimized. By applying the Chernoff bound to the conditional probability, we obtain

$$
\begin{gathered}
\operatorname{Pr}\left(A_{n}^{\lambda} \mid s_{1}, \ldots, s_{j}\right) \leq 2 e^{-\gamma \lambda} \cosh \left(\gamma c_{n}+\gamma \sum_{k=1}^{j} s_{k} d_{n k}\right) \\
\cdot \prod_{k=j+1}^{N} \cosh \left(\gamma d_{n k}\right)
\end{gathered}
$$

where the fact that the random variables $s_{j+1}, \ldots, s_{K}$ are independent and assume the values of 1 or -1 with equal probability has been used. Using the above analysis, a pessimistic estimator can be derived as

$$
\begin{aligned}
U_{n}\left(\lambda^{\star}, s_{1}, \ldots, s_{j}\right)= & 2 e^{-\gamma^{\star} \lambda^{\star}} \cosh \left(\gamma^{\star} c_{n}+\gamma^{\star} \sum_{k=1}^{j} s_{k} d_{n k}\right) \\
& \cdot \prod_{k=j+1}^{N} \cosh \left(\gamma^{\star} d_{n k}\right)
\end{aligned}
$$

for $j=1, \ldots, K$, where $\lambda^{\star}=\sqrt{2 \varepsilon \log (4 N)}, \gamma^{\star}=$ $\lambda^{\star} / \varepsilon$, and $\varepsilon=\max _{0<n<2 N-1}\left(c_{n}^{2}+\sum_{k=1}^{K} d_{n k}^{2}\right)$. By using (10) and (15), a suboptimal solution $s^{\star}$ for the problem in (6) can be obtained as

$$
\begin{aligned}
s_{j}^{\star}= & -\operatorname{sign}\left[\sum_{n=0}^{2 N-1} \sinh \left(\gamma^{\star} c_{n}+\gamma^{\star} \sum_{k=1}^{j-1} s_{k}^{\star} d_{n k}\right)\right. \\
& \left.\cdot \sinh \left(\gamma^{\star} d_{n k}\right) \prod_{k=j+1}^{K} \cosh \left(\gamma^{\star} d_{n k}\right)\right]
\end{aligned}
$$

for $j=2, \ldots, K$. Using (4b) and (16), the optimized OFDM symbol $\boldsymbol{X}^{\star}$ can be obtained as

$$
\boldsymbol{X}_{k}^{\star}= \begin{cases}X_{k} & \text { for } k \notin I \\ Y_{k}+s_{l}^{\star} Z_{k} & \text { for } k \in I\end{cases}
$$

where $l$ is the index of element $k$ in set $I$ in case that $k \in I$. It can be shown that the peak power of the optimized OFDM symbol $\boldsymbol{X}^{\star}$ is guaranteed to be smaller than $2 \lambda^{\star 2}$.

\section{B. Algorithm Based on Coordinate Descent Optimization}

Define $f(\mathbf{s})=\max \left\{f_{n}(\mathbf{s})\right.$ for $\left.0 \leq n \leq 2 N-1\right\}$ where

$$
f_{n}(\mathbf{s})=\left|c_{n}+\sum_{k=1}^{K} s_{k} d_{n k}\right| \quad \text { for } n=0, \ldots, 2 N-1
$$

The idea of CDO [8] can be applied to reduce the value of $f(\mathbf{s})$ iteratively where only one element of the sign vector $\mathbf{s}$ is allowed to switch in each iteration. First, the value of $f_{n}(\mathbf{s})$ after the sign switch of element $s_{k_{c}}$ can be obtained as

$$
f_{n}\left(\mathbf{s}, k_{c}\right)=\left|c_{n}+\sum_{k=1, k \neq k_{c}}^{K} s_{k} d_{n k}-s_{k_{c}} d_{n k_{c}}\right|
$$

for $n=0, \ldots, 2 N-1$ and $k_{c}=1, \ldots, K$. Let $\Delta_{f}$ be the change in the value of $f$, i.e.,

$\Delta_{f}\left(k_{c}\right)=f(\mathbf{s})-\max _{0 \leq n \leq 2 N-1} f_{n}\left(\mathbf{s}, k_{c}\right)$ for $k_{c}=1, \ldots, K$

and $\hat{k}_{c}$ be the index that yields the maximum of $\left\{\Delta_{f}\left(k_{c}\right), 1 \leq k_{c} \leq K\right\}$. If $\Delta_{f}\left(\hat{k}_{c}\right) \leq \epsilon$ where $\epsilon$ is a predefined tolerance, then a local minimum of function $f(\mathbf{s})$ is achieved and the algorithm terminates. Otherwise, the sign of $s_{\hat{k}_{c}}$ is switched and the sign vector can be updated as

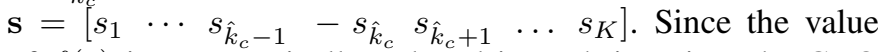
of $f(\mathbf{s})$ is monotonically reduced in each iteration, the CDO technique can be applied to enhance the performance of the MCP algorithms proposed in Secs. III.A and B.

\section{Combination of the Proposed and the SLM Algorithms}

In the proposed algorithms only one data set has been utilized for PAPR reduction. The performance can be improved by combining the proposed algorithms with the SLM algorithm, as illustrated in Fig. 3. First, multiple candidate data sets are generated at the transmitter. Second, for each of the data sets the proposed MCP algorithm is applied and the one with the least PAPR is selected. Third, the selective rotation (SR) [5] and CDO algorithms are applied to the data set selected in the second stage for further PAPR reduction.

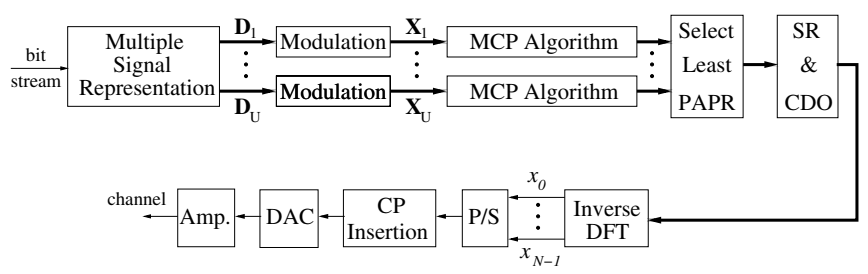

Fig. 3. Combination of the proposed and the SLM algorithms.

\section{Computational Complexity Analysis}

Note that compared with the scheme in Fig. 2c, the scheme in Fig. 2b requires fewer extended points for PAPR reduction. Statistically, the number of variables in (6) associated with the scheme in Fig. 2b is two thirds that associated with the scheme in Fig. 2c. Since the computation complexity of the MCP and $\mathrm{CDO}$ algorithms is propotional to the number of variables in 
problem (6), the computation required by the algorithms using the scheme in Fig. $2 b$ is only about two thirds that required by the algorithms using the scheme in Fig. 2c.

\section{Simulations}

The proposed algorithms were applied to an OFDM system with 64 subcarriers and the performance was evaluated and compared with that of the algorithms proposed in [4][5]. In order to approximate the analog signal accurately, oversampling was applied as in [5]. In the case where multiple candidate sequences are used, the number of sequences is denoted as $U$. In the case where the proposed MCP algorithm is combined with the SR algorithm, the number of rotations is denoted as $K$ and the rotation angles $\theta$ assume the values $\theta=0, \pi / K, \ldots,(K-2) \pi / K,(K-1) \pi / K$.

Example: Applying the proposed algorithms to the extended constellations in Fig. $2 \mathrm{~b}$ and $2 \mathrm{c}$, the clipping probabilities versus various power threshold values are plotted as the solid and dash curves in Fig. 4, respectively. For the SLM algorithm, a 16-QAM constellation was adopted. For the sake of comparison, the clipping probabilities obtained using the SLM algorithm and for the original OFDM signal are plotted in the same figure as dot-dashed curves. First, it can be observed from Fig. 4 that by combining the SR and the CDO algorithms with the MCP algorithm, significant PAPR reduction can be achieved over that obtained with the SLM algorithm. For example, for the MCP algorithm using the scheme in Fig. 2b with $K=4$ for a clipping probability of $10^{-3}$, a $0.5-\mathrm{dB}$ improvement can be achieved compared with the performance of the SLM algorithm with $U=16$. Second, it can be observed from Fig. 5 that the performance can be further improved by combining the proposed algorithms with the SLM algorithm. For example, a 1.5-dB improvement can be achieved by using the combined algorithm with $U=4, K=2$ over the SLM algorithm with $U=16$. Third, it can be observed from Figs. 4 and 5 that the performance of the algorithms using the scheme in Fig. $2 b$ is quite close to that of the algorithms using the scheme in Fig. 2c. However, the CPU time required by the former scheme is only about two thirds that required by the latter one.

\section{CONCLUSIONS}

Two new algorithms for PAPR reduction based on a new constellation extension scheme have been proposed. Simulations have demonstrated that the proposed algorithms outperform the SLM algorithm in [4] in terms of PAPR reduction and the algorithm in [5] in terms of computational complexity.

\section{ACKNOWLEDGEMENT}

The authors are grateful to Micronet, NCE Program, and the Natural Sciences and Engineering Research Council of Canada for supporting this work.

\section{REFERENCES}

[1] ETSI, "Radio broadcasting systems: digital audio broadcasting to mobile, portable and fixed receivers," European Telecommunication Standard, ETS 300-401, Feb. 1995.
[2] IEEE 802.11, "IEEE Standard for Wireless LAN Medium Access Control (MAC) and Physical Layer (PHY) Specifications," Nov. 1997.

[3] S. Mueller, R. Baeuml, R. Fischer, and J. Huber, "OFDM with reduced peak-to-average power ratio by multiple signal representation," Annals of Telecommunications, Feb. 1997.

[4] M. Breiling, S. Mueller-Weinfurtner, and J. Huber, "Distortionless reduction of peak power without explicit side information," Proc. IEEE GLOBECOM, pp. 1494-1498, 2000.

[5] Y. Kou, W.-S. Lu, and A. Antoniou, "New peak-to-average power-ratio reduction algorithm for OFDM systems using constellation extension," Proc. IEEE Pacific Rim Conference on Communications, Computers, and Signal Processing, pp. 514517, Victoria, BC, Aug. 2005.

[6] P. Raghavan, "Probabilistic construction of deterministic algorithms approximating packing integer program," J. Computer and System Sciences, vol. 37, pp. 130-143, 1988.

[7] J. Spencer, Ten Lectures on the Probabilistic Method, SIAM, Philadelphia, 1987.

[8] J. Luo, K. Pattipati, and P. Willett, "A class of coordinate descent methods for multiuser detection," Proc. IEEE ICASSP, vol. 5, pp. 2853-2856, Jun. 2000.

[9] J. G. Proakis, Digital Communications, 4th ed., McGraw Hill, 2000.

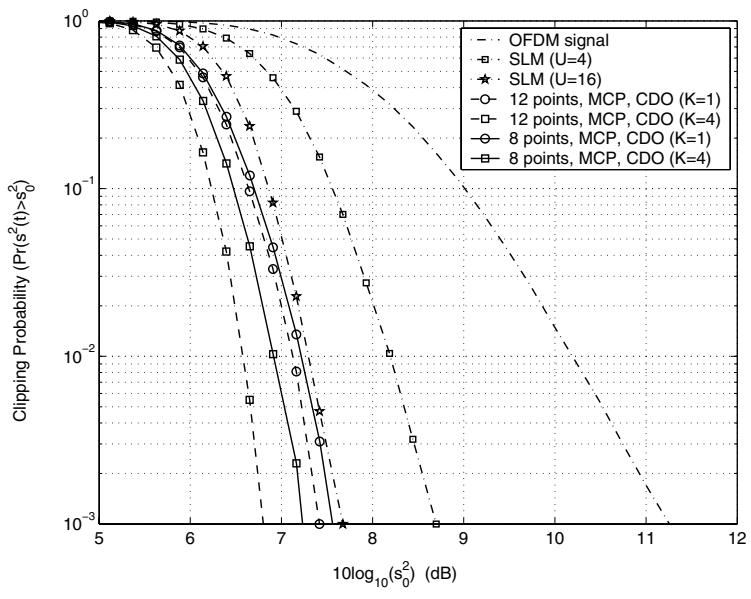

Fig. 4. Performance of the proposed algorithms.

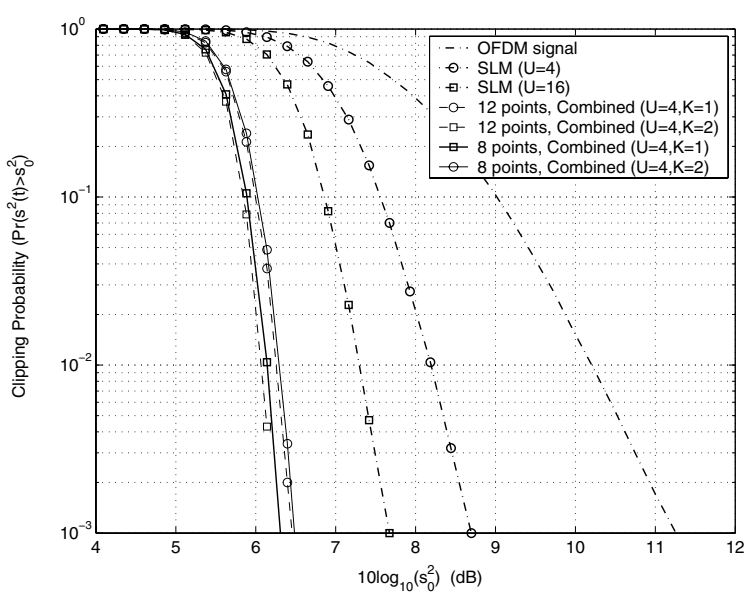

Fig. 5. Performance of the proposed algorithms which are combined with the SLM algorithm. 